\title{
Construyendo el perfil por competencias para el profesional en Educación Comercial
}

\author{
Building the Commercial Education Professional Competency Profile
}

\author{
Isabel Araya Muñoz' \\ Facultad de Ciencias Sociales \\ Escuela de Secretariado \\ Universidad Nacional \\ Heredia, Costa Rica \\ isaaraya@yahoo.com
}

Recibido 01 de marzo de 2012 • Corregido 09 de octubre de 2012 • Aceptado 07 de noviembre de 2012

Resumen. Este trabajo detalla las competencias del perfil por competencias para el profesional en Educación Comercial, producto del diagnóstico curricular realizado en la Carrera de Licenciatura en Educación Comercial, en el contexto de la Universidad Nacional de Costa Rica. La estrategia metodológica empleada en la realización del diagnóstico se basó en los principios de la investigación educativa. Para recabar información de las fuentes curriculares de la carrera en estudio: estudiantes de primer ingreso, estudiantes de licenciatura, profesionales en servicio y empleadores, se aplicaron encuestas escritas, previa validación de juicio de expertos y aplicación en una unidad de estudio. Se pretendía describir una situación educativa particular. La información se analizó a partir de dos grandes categorías: intenciones y principios, y alcance y desarrollo. Los resultados del diagnóstico se resumen en los rasgos que caracterizan al profesional de Educación Comercial, a saber: específicos de la disciplina, relativos a la gestión administrativa de la docencia, específicos y generales de la educación y de la pedagogía, y referentes al desarrollo humano. A partir de esos criterios, de los requerimientos de las fuentes curriculares y de las perspectivas curriculares de la Unidad Académica se concretaron ideas para la construcción de un perfil por competencias para este profesional. Las ideas que se proponen comprenden los fundamentos curriculares que basan el proyecto educativo en el que se plantea el perfil, conformado por: el objeto de estudio de la carrera, la competencia global o meta de formación, las competencias genéricas como ejes transversales y las competencias específicas, estas diferenciadas entre pedagógicas y disciplinares. Las competencias específicas propias de la disciplina se concentran en cuatro áreas de competencias: producción documental, soporte organizacional, recursos tecnológicos y administración de la información.

Palabras claves. Competencias, perfil profesional, perfil por competencias, Educación Comercial, secretariado, diagnóstico curricular.

\footnotetext{
1 Académica de la Escuela de Secretariado Profesional, Facultad de Ciencias Sociales de la Universidad Nacional de Costa Rica. Máster en Docencia Universitaria y en Planificación Curricular. Docente e investigadora.
} 
URL: http://www.una.ac.cr/educare

Abstract. This paper provides a complete description of the Commercial Education Professional Competency Profile that resulted from the curricular diagnosis of the Licenciatura en Educación Comercial, at the Universidad Nacional, Costa Rica. The methodological strategy used relies on the principles of research on education. Upon expert validation, written questionnaires were applied to first-year students, students of the licenciatura, practicing professionals and employers. The objective was to describe a particular education situation. Data was analyzed according to two categories: intentions/principles and scope/development. The findings resulted in the characteristics of the Commercial Education professionals, i.e. characteristics related to the discipline, characteristics related to the administrative management of teaching, specific and general characteristics of education and pedagogy, and characteristics associated to human development. Based on those criteria, on the curricular requirements of the information sources and on the curricular perspectives of the Academic Unit, ideas were put into practice to build the competency profile. The ideas proposed comprise the curricular fundamentals of the educational project on which the profile is set out, which include the subject of the study program, the global competency or training goal, the generic competencies as cross-cutting approaches, as well as the -pedagogical and disciplinary- specific competencies. The specific competencies of the discipline are focused on four competency areas: document production, organizational support, technological resources and information management.

Keywords. Competencies, professional profile, competency profile, Commercial Education, secretarial studies, curricular diagnosis.

\section{Introducción}

La exploración de nuevas alternativas que brinden al estudiantado un espacio pedagógico que le permita aprender para afrontar con sabiduría los problemas cotidianos de especialidad profesional es ocupación de la educación de hoy. Con esta consigna se realizó el diagnóstico de la carrera de Licenciatura en Educación Comercial de la Escuela de Secretariado Profesional y con base en esa idea se indagaron las condiciones contextuales para identificar la situación curricular.

La exploración de los diversos elementos curriculares partió de la pregunta: ¿Cuáles son los elementos que caracterizan el plan curricular de la carrera de Licenciatura en Educación Comercial de la Escuela de Secretariado Profesional de la Universidad Nacional?

Producto de esa etapa diagnóstica se obtuvo información de las fuentes curriculares de la carrera en estudio: estudiantes de primer ingreso, estudiantes de licenciatura, profesionales en servicio y empleadores; se aplicaron encuestas escritas, previa validación de juicio de expertos y aplicación en una unidad de estudio, al considerar que la intención era describir una situación educativa particular. La información se analizó a partir de dos grandes categorías: intenciones y principios, y alcance y desarrollo. 


\section{Resultados del diagnóstico}

A partir de la información recopilada en el diagnóstico curricular llevado a cabo en el contexto de la carrera de Licenciatura en Educación Comercial, se caracteriza la situación del plan curricular en los elementos básicos: contexto, recursos humanos, materiales, proceso educativo y demanda de profesionales. De esta esa manera se genera insumo para el establecimiento del perfil por competencias del profesional de esta carrera.

En términos generales, la gestión curricular de la Escuela de Secretariado Profesional cuenta con condiciones idóneas para el desarrollo de propuestas educativas innovadoras como la educación por competencias, se destacan aquí algunas:

- Calidad y suficiencia de las aulas, laboratorios de cómputo y de idiomas.

- Calidad y suficiencia de las aulas (iluminación, ventilación, tamaño, cantidad de mobiliario, comodidad, limpieza, aislamiento de ruidos y condiciones generales) que utiliza la carrera en el edificio del CIDE.

- Calidad y suficiencia de los laboratorios de cómputo (iluminación, ventilación, tamaño, cantidad de mobiliario, comodidad, limpieza, aislamiento de ruidos, cantidad de equipo, calidad del equipo, y condiciones generales) que se utilizan en la carrera en el edificio del CIDE.

- Servicio ofrecido por las bibliotecas de la Universidad Nacional (Biblioteca Joaquín García Monge (central), Centro de Documentación en Educación en el CIDE, CISCO, Facultad de Ciencias Sociales).

- Disponibilidad de redes de información académica (bibliotecas virtuales, revistas electrónicas, bases de datos, etc.) en el área de la Enseñanza de la Educación Comercial en el Sistema de Bibliotecas de la UNA.

- Disponibilidad de la bibliografía incluida en los programas de los cursos propios de la carrera en alguna de las bibliotecas de la Universidad Nacional.

- Enlaces de cooperación con empresas e instituciones para formación dual y para prácticas en las empresas.

- Oportunidades de proyección comunal por medio del Proyecto Manejo Básico de Oficinas, Proyecto de Formación Dual, colegios amigos, giras, práctica supervisada.

- Recursos tecnológicos: el aula virtual como medio para trascender las aulas.

- Servicio de becas y básicos en Vida Estudiantil.

- Servicios de Oficina de Registro, en forma personal y electrónica. 
De acuerdo con los aportes de las fuentes curriculares, los rasgos que caracterizan a un profesional en Educación Comercial se concretizan en conocimientos intelectuales y técnicos, específicos y generales, comportamientos humanos consigo mismo y con los otros. Se clasifican en disciplinares, administrativos o de gestión de la docencia, de la docencia y de desarrollo humano, según se detalla más adelante.

\section{Construyendo el perfil por competencias para el profesional en Educación Comercial}

Considerando los resultados del diagnóstico curricular realizado en el ámbito de la Escuela de Secretariado Profesional de la Universidad Nacional de Costa Rica, en el siguiente apartado se plantea una propuesta de rasgos que permiten la construcción de un perfil profesional por competencias para el profesional en Educación Comercial, el cual se orienta en una competencia global o meta de formación, las competencias genéricas y las competencias específicas, sobre la base de un proceso formativo fundamentado en los saberes esenciales: saber, saber hacer, ser y convivir; y en los ejes curriculares: integración disciplinaria, pedagogías contemporáneas y liderazgo académico y administrativo.

\section{Marco contextual}

La Escuela de Secretariado Profesional es una Unidad Académica de la Facultad de Ciencias Sociales de la Universidad Nacional y desde sus inicios, en 1974, se ha dedicado a formar profesionales en Secretariado Profesional y en Educación Comercial.

La carrera de Educación Comercial, vigente en la actualidad, tiene salidas de Diplomado, Bachillerato y Licenciatura, todas en idioma español y con fundamento en un modelo curricular por objetivos, en el que las áreas disciplinares tienen líneas de coordinación mínimas.

La gestión curricular de esta carrera la comparte la Escuela de Secretariado Profesional con la División de Educología, el Centro de Investigación, Docencia y Educación, y el Centro de Estudios Generales. La primera ofrece los cursos específicos de las ciencias secretariales; la segunda, los cursos del área de educación y, el tercero, los cursos de desarrollo humano en el área de cultura, ciencia y tecnología. Este trabajo curricular se realiza, en la actualidad, únicamente con la coordinación administrativa; por otro lado, cada ente planifica y organiza desarticuladamente.

La Escuela de Secretariado Profesional, como institución educativa pública del nivel superior, tiene la misión de formar personas en forma integral, como se establece en su misión:

Formar integralmente profesionales de excelencia en el área del secretariado y/o [sic] la administración de oficinas en los ámbitos de la gestión administrativa, empresarial y educativa, atendiendo a [sic] las demandas de la sociedad para contribuir al desarrollo humano sostenible. (Universidad Nacional de Costa Rica, Escuela de Secretariado, 2007, p. 4) 
De acuerdo con ese cometido, la Escuela de Secretariado Profesional, a partir del 2011, se dedica a la formación integral de futuros profesionales mediante la formación por competencias. De esta manera pretende un rediseño curricular como propuesta de innovación educativa, cuyo propósito es lograr procesos de desarrollo de capacidades para adaptar a situaciones imprevistas y poder dar respuestas reflexivas en lugar de responder con rutinas y acciones prescritas.

En razón de lo anterior, se plantea a continuación la definición del objeto de estudio que enmarca la profesión de Educación Comercial y los fundamentos curriculares que caracterizan el contexto del perfil que se propone más adelante.

\section{Objeto de estudio}

Para esta propuesta, y producto de la opinión de las fuentes curriculares, se plantea la definición del objeto de estudio de la Educación Comercial como la promoción de espacios pedagógicos para el desarrollo de competencias orientadas a la gestión de apoyo administrativo, en las empresas públicas, privadas y mixtas, y de toda índole productiva, en las áreas de servicios, de comercio, agrícolas, profesionales, industriales, educativas; en fin, en todas las áreas económicas de un país, de una región y del mundo.

Este objeto integra las dimensiones de la disciplina Educación Comercial, cuyos espacios hacia otras ciencias y disciplinas que la sustentan son: Desarrollo humano, Comunicación en inglés y en español, Secretariado, Psicología, Educación, Investigación, Cultura general, Derecho, Archivística, Tecnológicas de la información y la comunicación y Gestión empresarial.

En el marco del objeto de estudio de la carrera de Educación Comercial se plantean los aspectos filosóficos, epistemológicos y psicopedagógicos que fundamentan la propuesta de rediseño curricular por competencias.

\section{Fundamentos curriculares}

Con el propósito de establecer el perfil profesional por competencias, es necesario evidenciar los fundamentos filosóficos, epistemológicos y psicopedagógicos de la carrera de Licenciatura en Educación Comercial en el ámbito en estudio.

El fundamento filosófico de un proyecto formativo, que responda al crecimiento integral de un profesional, se establece en los cuatro pilares de la educación, los cuales Delors (1996) define como: el aprender a conocer, el aprender a hacer, el aprender a vivir y el aprender a ser. Estos principios prevalecen en los fines de la educación costarricense y en los diversos niveles estratégicos de la institución en la que se enmarca la propuesta. Se establece la filosofía de formar profesionales competitivos y con compromiso social, como un individuo íntegro y que forma parte de una sociedad en la cual desarrolla su potencial en un puesto de trabajo en 
URL: http://www.una.ac.cr/educare

un mundo competitivo y cambiante y que, a la vez, cultiva los valores de la solidaridad y la colaboración que le permitan ser un ciudadano para la vida en comunidad y en libertad.

Los fundamentos epistemológicos de la enseñanza de una especialidad pedagógica como la educación comercial consideran la integración de la teoría y la práctica reflejada en determinados propósitos, perspectivas, experiencias, valores e intereses. Torres (1998) indica que las personas construyen sus explicaciones o interpretaciones por medio de actividades de aprendizaje propias de enfoques pedagógicos contemporáneos. Esto se logra porque la educación centrada en la persona tiene como propósito transformar la realidad.

Las fuentes psicopedagógicas, en un proceso de formación profesional, comprenden el aporte de la psicología y de la pedagogía que orienta el proceso de enseñanza y de aprendizaje en la interacción teoría-práctica con fundamento en principios de autonomía del individuo. Desde esa concepción y de acuerdo con Tobón (2005), una estructura curricular considera factores como: una meta educativa que guía hacia la resolución de problemas de la realidad; la dinámica pedagógica se establece en la aplicación de principios pedagógicos contemporáneos (Cerezo, 2006) como trabajo colaborativo, incorporación de nuevas tecnologías, resolución de casos, elaboración de actividades en ambiente simulado, gestión de proyectos, actividad experiencial, indagación y trabajo autónomo y colaborativo, así como encuentros pluriculturales.

Las competencias del docente en Educación Comercial deben ser desarrolladas en un ámbito congruente con principios integradores en la formación, por tanto, siguiendo ideas de Hernández (1988) el énfasis en un modelo de diseño curricular con perspectivas de educación centrada en la persona y en la participación social son pertinentes a la hora de planificar el currículum. En tal sentido se consideran los siguientes factores para orientar las estrategias de enseñanza y de aprendizaje:

- Docentes y estudiantes son protagonistas de las actividades de aprendizaje en la experiencia de aula o fuera de ella.

- El proceso de construcción del aprendizaje se fundamenta en el liderazgo académico, en la actitud e interés del estudiante y en los recursos disponibles.

- Prevalece el sentido de innovación, a partir de la deliberación de sus participantes.

- La orientación metodológica se centra en las características propias de la persona, cómo aprenden, cómo desarrollan sus capacidades, cuáles son las habilidades para conseguir información, para comunicarse, para comprender el entorno.

Estas ideas se relacionan con teorías pedagógicas contemporáneas (Cerezo, 2006), ya que se parte de la realidad del alumno, de sus conocimientos previos y de su experiencia, vivencias y valores, aprendizaje en libertad y su meta es la transformación de la persona. 
En resumen, los fundamentos curriculares, desde proyectos educativos conducidos por el modelo de desarrollo de competencias, tienen sustento en tres premisas: el qué, el cómo y el para qué de la educación. Consideran que el proceso formativo se postula como un proceso de enseñar no solo para un puesto de trabajo, sino para el desarrollo de la persona en todos sus potenciales; por tanto, el fundamento filosófico se enmarcó en los fines más esenciales de la educación: educar para la transformación de la persona y de la sociedad; el fundamento epistemológico coloca a la persona como centro de un proceso formativo; y el fundamento psicopedagógico establece las interacciones del contexto y de los actores del proceso educativo para el desarrollo de las competencias.

Estos fundamentos se enfocan en el modelo de diseño curricular, de corte práctico deliberativo y crítico (Bolívar, 2000), con una perspectiva de la educación dentro de las líneas de los paradigmas pedagógicos contemporáneos, donde se considera a la persona estudiante como una persona activa y a la persona docente como generador de procesos de enseñanza y de aprendizaje.

\section{Marco conceptual}

En este apartado se consideran algunos elementos que se definen en torno a la construcción de un perfil profesional por competencias, tales como perfil profesional, sus ejes curriculares, la competencia global o meta de formación, competencias genéricas y competencias específicas.

\section{Perfil profesional}

El perfil profesional por competencias corresponde a la representación integral de logros o metas de formación de un proyecto curricular; esos rasgos se concretan en la formación de la persona y del profesional, quien una vez terminada su carrera universitaria pueda servir a la sociedad, por tanto, se constituye por los conocimientos intelectuales, procedimientos técnicos específicos y generales, comportamientos humanos consigo mismo y con las demás personas.

Esos saberes deben proporcionar una formación básica tanto teórica como práctica, que le permita a la persona aplicarla a distintos entornos del área profesional y para la vida. En ese sentido, el perfil es producto de las condiciones que imperan en el currículo en un momento dado.

El perfil profesional, como una idea que guía los procesos formativos, tendrá el resultado de un profesional competente que puede aplicar esas competencias en el ámbito profesional y en otras situaciones de la vida. Por eso se definen cuatro elementos que se consideran integradores del perfil profesional: la competencia global o meta de formación, los ejes curriculares, las competencias genéricas y las competencias específicas. Estos rasgos derivan una serie de módulos o proyectos formativos por áreas competenciales, según la propuesta 
URL: http://www.una.ac.cr/educare

de la carrera en estudio. En este sentido, Ricoy-Lorenzo y Pino-Juste (2005) consideran que, al ingresar a un proceso educativo, es necesario explicitar los requisitos administrativos y la relación de contenidos que deben reunir los estudiantes. Estos requerimientos deben ser congruentes con el programa formativo y ser accesibles y públicos.

En cuanto al perfil de egreso, también denominado perfil de salida o perfil del graduado, Quesada, Cedeño y Zamora (2001) lo definen el perfil de graduado como el conjunto de competencias que debe poseer el egresado al finalizar la carrera.

Es importante diferenciar que, en el contexto de la Universidad Nacional, el concepto de egresado corresponde a la persona estudiante que ha aprobado todos los créditos de la carrera en los diferentes grados; el graduado es la persona que ha aprobado el requisito del Trabajo Final de Graduación, de acuerdo con el plan de estudios respectivo, y ha realizado su proceso de juramentación correspondiente.

Hawes y Corvalán (2005) mencionan que los conceptos del perfil profesional están relacionados con el contexto laboral en una época y sociedad dadas, la organización del trabajo, el grado de autonomía del ejercicio profesional y las relaciones entre las diferentes ocupaciones que afectan su especificidad. En ese contexto, define el perfil profesional para el nivel del egresado, el "(...) profesional inicial básico" (p. 13), es decir, el profesional recién graduado. Diferencia el perfil del "(...) profesional experto" (p. 13) como la caracterización de una persona que tiene su título profesional y una vasta experiencia en un puesto de la especialidad.

Por su parte, Alfaro et al. (2008) conceptualizan el perfil de desempeño en la relación de características personales y conductuales, cuya valoración la realizan por medio de "(...) la diversidad, la diferenciación de particularidades humanas, las capacidades, los valores, las actitudes, los estilos cognitivos y las pautas de comportamiento" (pp. 39-40). Aunque no diferencian los conceptos, son específicos al definir el perfil docente como rasgos específicos que caracterizan al educador y que pueden convertirse en un orientador para la selección y la contratación laboral, la calificación, la evaluación y la promoción docente.

También en el ámbito educativo, Rodríguez (2010) menciona la conceptualización del perfil docente en su trabajo para puntualizar "(...) el perfil del docente de matemática (...) desde la tríada matemática-cotidianidad- y pedagogía integral (...)" (p. 2). Al respecto indica que el perfil docente se basa en especificaciones sobre el hacer y el compromiso con la realidad educativa.

Hernández (2004) considera prudente tener en cuenta varias dimensiones a la hora de definir un perfil profesional, la persona humana, intelectual, profesional, social, y su desempeño operativo. En ese sentido, concibe el perfil profesional como una imagen previa de las características, los conocimientos, las habilidades, los valores y sentimientos que debe haber desarrollado el estudiante en su proceso de formación. 
Desde el enfoque por competencias, también se ha conceptualizado el perfil profesional. Bozu (2007) ha diferenciado el perfil profesional del perfil competencial del profesorado como una descripción detallada y esmerada que muestre los rasgos más característicos de un grupo profesional, es decir" (...) el conjunto de capacidades y competencias que identifican la formación de una persona, para asumir en condiciones óptimas las responsabilidades propias del desarrollo de funciones y tareas de su profesión (p. 3).

Desde la formación profesional, en el nivel universitario público, es relevante anotar que la caracterización de las personas que concluyen su formación académica para acercarse a las oportunidades laborales y su desempeño y convivencia en la sociedad, en todos sus ámbitos, en cualquier enfoque educativo que se plantee, deben responderse dos preguntas básicas: ¿qué tipo de ciudadano requiere el país?, ¿cuáles son las características que lo califican como persona y como profesional? En ese sentido, Araya (2011) plantea:

(...) es importante reflexionar sobre los fines, metas o ideas de la educación, concretizada en un proyecto educativo, cuya orientación es el aprender, es decir, ¿qué aprender?, ¿cómo aprenderlo? y ¿para qué aprenderlo? Las respuestas dependen del significado de la educación. En tal sentido Kemmis (1998) indica que desde la aparición de la educación de masas, la planificación curricular ha tratado de transformar la sociedad mediante cambios en los currículos y en la escolarización, por ello el currículo no debe considerarse solo como la organización de lo que debe ser enseñado y aprendido. (p. 113)

Considerando las propuestas anteriores, el perfil profesional se admite como la representación de una propuesta curricular, que se concreta en la integralidad de la persona y del profesional, quien una vez terminada su carrera universitaria pueda servir a la sociedad; por tanto, en ese proceso educativo se integran conocimientos -intelectuales y técnicos, específicos y generales- y comportamientos humanos consigo mismo y con los demás.

Esa integración de saberes debe proporcionar a la persona una formación básica sólida, tanto teórica como práctica, que le permita su aplicación en distintos entornos del área profesional y para la vida; esto es, el perfil profesional es producto de las condiciones que imperan en el currículo en un momento dado.

\section{Ejes curriculares}

Los ejes curriculares son elementos intencionales del diseño curricular y que, en la realidad actual de la Universidad Nacional, aportan a la gestión académica, de acuerdo con las corrientes pedagógicas contemporáneas, las exigencias de calidad y eficiencia en los profesionales, así como elementos de desarrollo humano y profesional de académicos y administrativos. 
En los ejes curriculares se asienta el cambio de modelo pedagógico, al crearse una dinámica de gestión académica y administrativa. Por eso se plantean como ejes curriculares fundamentales para el desarrollo de un proceso educativo, orientadores del desarrollo de las competencias del perfil profesional en Educación Comercial. Estos son: a) Integración disciplinaria, b) Liderazgo académico y liderazgo administrativo y c) Pedagogías contemporáneas.

La integración del currículo, desde el punto de vista disciplinar y de acuerdo con Torres (1998), es la oportunidad de analizar el proyecto educativo en la gestión del conocimiento, por medio de acciones conjuntas hacia una misma meta formativa. En el contexto de la Escuela de Secretariado Profesional, constituye una propuesta para la articulación multidisciplinaria en la que las disciplinas mantienen su integridad; se seleccionan temas para desarrollar en unidades de forma multidisciplinaria, se exige trabajo en equipo; cada disciplina aporta al estudio del tema respondiendo en forma asertiva las demandas de la sociedad del conocimiento, en donde el desarrollo de saber holístico centraliza el proceso formativo.

El liderazgo académico y el liderazgo administrativo, al igual que el eje de Pedagogías Contemporáneas, como se anotó en los fundamentos curriculares, responden a las cuestiones filosóficas, epistemológicas y psicopedagógicas que basan un proyecto formativo, por tanto, remiten a criterios de asunción de los deberes y obligaciones de la persona docente, de la persona estudiante y de la persona administradora curricular para proveer una estructura que posibilite el logro de las metas educativas, a través de trabajo interdisciplinario, excelencia en el desempeño, profesionalización docente y administrativa, capacitación permanente, disposición y adaptación a los cambios vertiginosos en la ciencia y las tecnologías de la comunicación y de la información, a los nuevos sistemas productivos y los cambios culturales que se generan de los procesos de movilidad social y territorial.

Los tres ejes son complementarios y no es posible aplicar uno de ellos en forma independiente, por eso, el compromiso es involucrar a todos los que integran la organización.

\section{Competencia global o meta de formación}

La competencia global o meta de formación es una orientación hacia el desarrollo que delimita los diferentes módulos curriculares, sus competencias específicas y genéricas. Cada persona tiene un desarrollo competencial particular, debido a sus propias capacidades, sus necesidades, intereses y condiciones específicas.

Esta idea de generar el proceso de desarrollo de las competencias profesionales a partir de una competencia global o meta de formación parte de las propuestas de Tobón (2005), quien basa la estructura de competencias profesionales en una competencia global planteada a partir 
de nodos problematizadores. La competencia global o meta formativa involucra los propósitos de un proyecto educativo que se vislumbra en el perfil profesional por competencias para el profesional en Educación Comercial; integra los conocimientos intelectuales, procedimientos técnicos, administrativos y legales; destrezas y habilidades pertinentes, actitudes necesarias para el logro de resultados con eficiencia y calidad, fundamentado en principios de desarrollo humano.

\section{Competencias genéricas}

Las competencias genéricas como ejes transversales que fortalecen el currículo en cuanto a actitudes, valores y comportamientos. Son intenciones para enriquecer el currículo.

En el ámbito del Proyecto Tuning América Latina, Badilla (s. f.), se refieren las competencias genéricas a los conocimientos generales para realizar comportamientos laborales y habilidades que empleen tecnología. Para alcanzarlas, es ineludible la coherencia entre los programas curriculares, el desempeño natural y el trabajo real de ese profesional en el ámbito local, nacional e incluso internacional.

Las competencias genéricas también son referidas a los comportamientos asociados con el desempeño, común a diversas ocupaciones y ramas de la actividad productiva, debido a la gran variedad de comportamientos, actitudes y disposiciones necesarias para el desarrollo humano. Es importante para todo proyecto formativo una definición contextual de estas competencias, así como su aplicación en los diversos niveles de la malla curricular, por tanto, su conceptualización y aplicación específica va a depender de la deliberación del cuerpo académico y administrativo en la programación curricular y en su puesta en práctica, así como de la importancia de integrar los cursos de cultura, ciencia, tecnología y desarrollo humano que brinda el Centro de Estudios Generales de la Universidad Nacional para complementar la carrera.

En el ámbito de la Universidad Nacional, Araya y Varela (2011) realizaron un estudio de las 27 competencias genéricas seleccionadas por el Proyecto Tuning América Latina, las cuales, según Beneitone et al. (2007), fueron escogidas de un listado de 85 que, académicos, estudiantes, graduados y empleadores entre 19 países y 190 universidades latinoamericanas de 12 áreas temáticas o carreras universitarias comunes, consideraron con mayor grado de importancia y de realización de cada una.

Esas 27 competencias genéricas, Araya y Varela (2011) las relacionaron con los principios del Modelo Pedagógico de la Universidad Nacional y las sometieron a consulta sobre el grado de importancia, a académicos involucrados en proyectos afines con el tema de competencias, así como con procesos de acreditación de carreras. De esta forma, eligieron las ocho con mayor puntaje y las aplicaron en el estudio referido. Son las siguientes: 
URL: http://www.una.ac.cr/educare

Tabla 1

Competencias genéricas

Capacidad de trabajo en equipo

Compromiso con la calidad

Capacidad para identificar, plantear y resolver problemas

Compromiso ético

Capacidad para actuar en nuevas situaciones

Capacidad de aprender y actualizarse

Capacidad creativa

Capacidad crítica y autocrítica

Nota: Competencias genéricas definidas por Araya y Varela (2011) en el contexto de la Escuela de Secretariado Profesional de la Universidad Nacional

Estas ocho competencias genéricas sirven de referencia para orientar el análisis del perfil del profesional en la carrera en estudio.

\section{Competencias específicas}

Las competencias específicas identifican los comportamientos asociados a conocimientos técnicos de una tarea. En el marco del Proyecto Tuning, Badilla (s. f.) se refiere a las competencias específicas con conocimientos especializados para realizar labores concretas propias de una profesión o disciplina, que se aplican en determinado contexto laboral, tal sería el caso de la relación con pacientes o la elaboración de estados financieros.

Por su parte, Rial (2007) también define las competencias específicas como las propias de una ocupación o profesión determinada y singular. Se caracterizan por tener un alto grado de especialización y comprender procesos educativos específicos, generalmente llevados a cabo en programas técnicos de formación para el trabajo y la educación superior.

Considerando esas propuestas, las competencias específicas se determinan como los conocimientos técnicos y procedimentales, y las habilidades y destrezas concretas de una profesión o disciplina orientadas a un contexto específico y que pueden ser adaptadas a situaciones relacionadas. 


\section{Metodología para la elaboración del perfil}

La metodología utilizada para elaborar el perfil consistió en tomar las categorías resultantes del diagnóstico curricular, en cuanto a rasgos que caracterizan a la persona profesional en Educación Comercial. Se clasificaron en seis grandes apartados competenciales: Producción documental, soporte organizacional, recursos tecnológicos, administración de la información, docencia y genéricas. Cada uno de estos sitúa los saberes fundamentales para el desarrollo de las competencias profesionales: saber, saber hacer, saber ser y saber convivir. En esa sistematización, cada área competencial se catalogó en el campo disciplinar que apoya la solución de los problemas o necesidades.

Estos apartados competenciales también se complementaron con el insumo generado en el diagnóstico de parte de empresas públicas autónomas, la Dirección General del Servicio Civil de Costa Rica (2009) y una empresa privada en cuanto a funciones de puestos secretariales en oficinas de más alta jerarquía en la institución.

A partir de esa sistematización se derivaron los siguientes elementos que caracterizan al profesional en el ámbito ocupacional:

- Rasgos específicos de la disciplina del secretariado

- Rasgos relativos a gestión administrativa o de gestión de la docencia

- Rasgos propios de la docencia

- Rasgos relativos al desarrollo humano

Estos rasgos integran las competencias específicas que, como se indicó anteriormente, se clasificaron en competencias disciplinares y competencias pedagógicas.

Para complementar esos requerimientos ocupacionales en la propuesta de perfil se integran: una competencia global o meta de formación, los ejes curriculares, las competencias genéricas, y el sustento de un enfoque curricular orientado al desarrollo de la persona en su potencial y en torno a las necesidades comunales; así como los paradigmas pedagógicos contemporáneos que consideran al sujeto como una persona activa y a la persona docente como generadora de procesos de enseñanza y de aprendizaje.

\section{Componentes del perfil}

El perfil profesional por competencias del profesional en Educación Comercial integra los componentes que dan soporte a un proyecto que pretende formar personas hacia el desarrollo personal y profesional. Esos componentes se esquematizan en la figura 1 que incluye: una competencia global o meta de formación, los ejes curriculares, 
las competencias específicas y genéricas. Estos elementos se sustentan en un enfoque curricular orientado hacia desarrollo de la persona en su potencial y en torno a las necesidades comunales; $y$ en los paradigmas pedagógicos contemporáneos, al considerar el sujeto como una persona activa y a la persona docente como generadora de procesos de enseñanza y de aprendizaje.

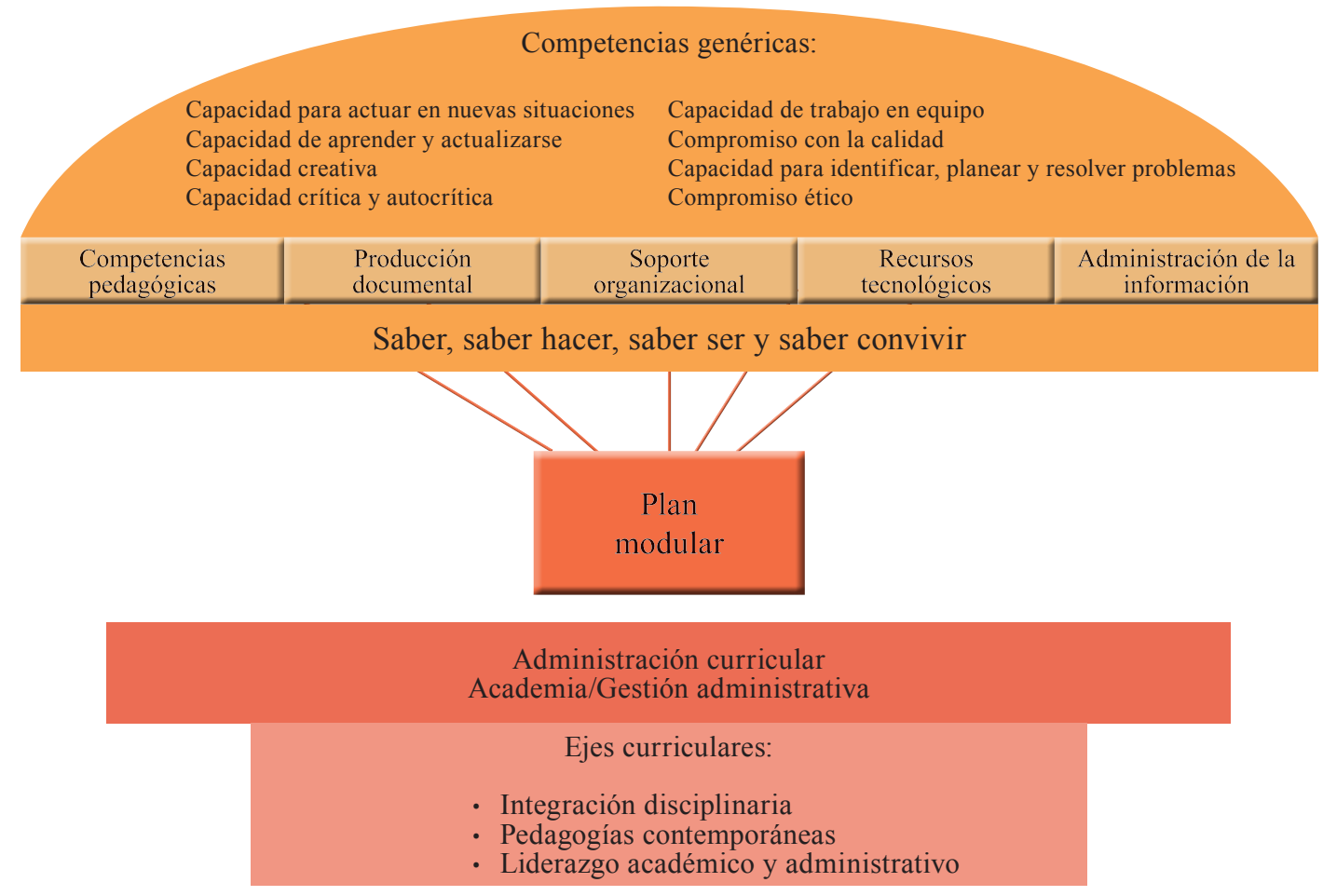

Figura 1. Componentes del perfi profesional por competencias para el profesional en Educación Comercial

\section{Competencia global o meta de formación}

El profesional en Educación Comercial es competente para generar procesos de enseñanza y de aprendizaje del Secretariado o Administración de Oficinas, en los diferentes contextos públicos y privados y en los diferentes niveles educativos: técnico, parauniversitario y universitario, de acuerdo con los principios de desarrollo humano y de las competencias requeridas en el ámbito de apoyo organizacional. 


\section{Competencias genéricas}

1. Capacidad de trabajo en equipo

2. Compromiso con la calidad

3. Capacidad para identificar, planear y resolver problemas

4. Compromiso ético

5. Capacidad para actuar en nuevas situaciones

6. Capacidad de aprender y actualizarse

7. Capacidad creativa

8. Capacidad crítica y autocrítica

\section{Competencias específicas}

\section{Pedagógicas}

1. Reconocer la historia, los principios y los fines de la educación costarricense como medio de desarrollo humano y de transformación social y su relación con la Educación Comercial.

2. Comprender los alcances del currículo educativo y su abordaje desde las diversas perspectivas sociales.

3. Desarrollar capacidad crítica, autocrítica y reflexiva para atender diversas propuestas pedagógicas y relacionarlas con la realidad educativa de la persona estudiante.

4. Reconocer los principios y las perspectivas pedagógicas que orienten procesos de enseñanza y de aprendizaje para la formación de personas competentes en su campo profesional, con fundamento en valores humanos para la convivencia con otros y otras.

5. Comprender las condiciones contextuales de la persona estudiante para diseñar escenarios, procesos y experiencias de aprendizaje significativo y relevante, en torno a la especialidad de Secretariado o Administración de Oficinas y a su contexto profesional.

6. Generar procesos de enseñanza y de aprendizaje del Secretariado y Administración de Oficinas con base en las necesidades educativas de la persona estudiante y de acuerdo con la integración de saberes. 
7. Orientar, guiar y acompañar el trabajo y la indagación de datos que le permitan a la persona estudiante desarrollar la comprensión teórica para aplicarla a la resolución de los problemas de su realidad.

8. Participar con liderazgo académico en actividades que coadyuven al desarrollo integral de la persona estudiante en el ámbito institucional, nacional e internacional.

9. Investigar, en forma permanente, la realidad educativa de la Educación Comercial que permita la mejora continua de los procesos de enseñanza y de aprendizaje.

10. Crear propuestas de desarrollo didáctico de la Educación Comercial, sustentadas en el desarrollo de competencias de la persona estudiante por medio de integración de saber, saber hacer, saber ser y saber convivir.

11. Proponer innovaciones al utilizar recursos tecnológicos en los proyectos educativos de Educación Comercial.

12. Participar, de forma proactiva y propositiva, en actividades de análisis y en propuestas de mejora de proyectos curriculares en el ámbito institucional, nacional o internacional.

13. Atender, de acuerdo con los requerimientos institucionales, la instrumentalización relacionada con la gestión pedagógica y didáctica de la Educación Comercial.

14. Aplicar los recursos tecnológicos en los procesos pedagógicos y en la gestión administrativa de la docencia.

\section{Disciplinarias}

Área competencial: producción documental

1. Integrar los conocimientos intelectuales, procedimientos técnicos, administrativos y legales sobre las características internas y externas para producir los diferentes tipos de documentales, sencillos y complejos, que se requieren en las oficinas y en la docencia, en español y en inglés.

2. Aplicar las herramientas tecnológicas requeridas para producir los diferentes tipos documentales, sencillos y complejos, que se requieren en las oficinas y en la docencia, en español y en inglés.

3. Aplicar, con actitud crítica y reflexiva, las condiciones técnicas y humanas en la producción documental orientada a la calidad de vida de la persona y a la conservación del ambiente. 
Área competencial: soporte organizacional

1. Integrar los conocimientos intelectuales, procedimientos técnicos, administrativos y legales para atender requerimientos de apoyo en trámites de gestión del cliente, de procesos, de recursos materiales y humanos en las oficinas, en español y en inglés.

2. Orientar el servicio hacia la resolución de problemas de apoyo a la organización con principios científicos, con liderazgo profesional, con calidad y con fundamentado en valores humanos para la convivencia.

Área competencial: recursos tecnológicos

1. Integrar conocimientos intelectuales, procedimientos técnicos, administrativos y legales sobre la aplicación de recursos tecnológicos especializados en la gestión de apoyo en la oficina y en la docencia.

2. Aplicar, con actitud crítica y reflexiva, los recursos tecnológicos especializados para la gestión de apoyo en la oficina.

3. Aplicar, con actitud crítica y reflexiva, los recursos tecnológicos especializados para la gestión pedagógica de la Educación Comercial.

\section{Área competencial: administración de la información}

1. Integrar conocimientos intelectuales, procedimientos técnicos, administrativos y legales para la administración de la información en la oficina y en la docencia.

2. Aplicar, con actitud crítica y reflexiva, la administración de la información en las oficinas, de acuerdo con las innovaciones tecnológicas y los principios de seguridad de la información y manipulación de datos privados.

\section{Ejes curriculares}

En los ejes curriculares se inscribe el cambio de modelo pedagógico, cuya base se centra en una gestión académica y administrativa sustentada en los siguientes ejes curriculares:

El primer eje corresponde a la integración disciplinaria. Este se operacionaliza con el criterio de la articulación multidisciplinaria. Las disciplinas mantienen su integridad, se seleccionan temas para desarrollar en unidades de forma multidisciplinaria, exige trabajo en equipo, cada disciplina aporta al estudio del tema.

El segundo eje es el liderazgo académico y liderazgo administrativo. Este eje integra la labor de dos personajes. La del primero se describe con los criterios de una persona 
académica comprometida con la profesión, autónoma, propositiva, proactiva, con formación especializada en el área disciplinar y en el área pedagógica, motivada por los fines, principios y objetivos propuestos por la Unidad Académica y la universidad pública costarricense. Desde el ámbito de la gestión o administración curricular, los criterios que prevalecen son los de coordinación mano a mano de la gestión educativa; de generación de enlaces de cooperación a lo interno de la organización para el logro de las metas educativas y de favorecimiento del diálogo, la deliberación y el consenso con diversos promotores del proceso educativo.

El tercer eje lo constituyen las pedagogías contemporáneas. Motiva los dos anteriores y se centra en los criterios de situaciones de aprendizaje autónomas, responsabilidad de los estudiantes en su propio desarrollo, el consenso entre los involucrados, investigación acción-reflexión para estudiar e intervenir la realidad para transformarla e interacción escuelacomunidad en el estudio de las necesidades individuales, formativas y de intervención social.

Las competencias genéricas. Se tomaron de las ocho competencias específicas que Araya y Varela (2011) seleccionaron a partir del escrutinio que académicos y administrativos de la Universidad Nacional realizaron del listado de propuestas por el Proyecto Tuning. Estas competencias fueron cotejadas con los principios del modelo pedagógico de la Universidad Nacional y con las necesidades planteadas por las fuentes curriculares. Son parte del perfil profesional con criterio de transversalidad, ya que cruzan el currículo en el proceso, en las áreas de desarrollo personal y para la convivencia en la sociedad. Deben ser definidas de acuerdo con el análisis, la deliberación y el consenso del equipo de académicos y gestores educativos.

La operacionalización de los ejes curriculares se visualiza en la integración administrativa y académica de las dos unidades académicas responsables de la carrera de Educación Comercial.

\section{Validación del perfil}

La validación de un perfil por competencias comprende la confirmación de la vigencia de los rasgos propuestos, de acuerdo con las intenciones y principios del proyecto educativo. En ese sentido, Hawes y Corvalán (2005) proponen al menos cuatro modos de validación, los cuales están en función de la audiencia, los intereses y las vías: académicos y gremios, postulantes y sus familias, mercado laboral, sociedad en general (agencias de financiamiento o acreditación).

En este caso se realizó la validación de parte de académicos y académicas de la Escuela de Secretariado Profesional, por medio de una encuesta escrita que detalla cada una de las competencias genéricas y específicas. Se le pidió a cada académico que valorara el nivel de pertinencia de cada competencia, así como su percepción de todas ellas en relación con los siguientes argumentos:

- Delimitación de los dominios de competencias.

- Descripción de los dominios de competencias (grupos de capacidades y tareas esenciales relacionadas que deben ser desplegadas por el futuro profesional). 
- Determinación de la pertinencia, la relevancia y la congruencia de los rasgos: en el ámbito social, económico, político de la unidad académica.

- Congruencia con el modelo pedagógico de la Universidad Nacional

- Tendencias de la profesión emergentes, decadentes y operantes.

- Existencia de una relación entre los elementos del perfil y a misión de la Escuela de Secretariado Profesional.

- Acogida de los principios filosóficos, epistemológicos y psicopedagógicos de la Universidad Nacional.

- Evidencia de incorporación de los avances científicos y tecnológicos.

- Posibilidad de empleabilidad del graduado con esos rasgos.

- Rasgos que responden a las nuevas exigencias del mercado laboral y a las de una persona formada integralmente para la sociedad actual.

El equipo de académicos que participó en la validación corresponde a ocho personas que se encuentran participando en los talleres de capacitación en el tema de competencias que la Escuela de Secretariado Profesional está desarrollando desde junio de 2011. Fueron escogidos para la validación del perfil considerando dos criterios: el conocimiento que tienen sobre competencias en educación y la experiencia docente en la Escuela de Secretariado Profesional.

Los resultados de la validación se detallan en las tablas 2 y 3.

Tabla 2

Perfil por competencias para la persona profesional en Educación Comercial Escala de valoración de la pertinencia

\begin{tabular}{lccc}
\hline & \multicolumn{2}{c}{ Grado de pertinencia } \\
\cline { 2 - 4 } & Alto & Medio & Bajo \\
\hline $\begin{array}{l}\text { Competencias genéricas } \\
\text { Competencias específicas }\end{array}$ & $95 \%$ & $3,1 \%$ & $0 \%$ \\
$\quad$ Pedagógicas & & & \\
$\begin{array}{l}\text { Disciplinarias } \\
\text { Área competencial: producción documental } \\
\text { Área competencial: soporte organizacional }\end{array}$ & $61 \%$ & $28 \%$ & $1 \%$ \\
$\quad$ Área competencial: recursos tecnológicos & $75 \%$ & $25 \%$ & $0 \%$ \\
Área competencial: administración de la información & $75 \%$ & $17 \%$ & $0 \%$ \\
\hline
\end{tabular}

Nota: Resultados de validación, promedios porcentuales, según grado de pertinencia seleccionado por la muestra 
URL: http://www.una.ac.cr/educare

Según los promedios porcentuales que arroja la tabla 2, el listado de competencias que conforman el perfil por competencias para el profesional en Educación Comercial, desde la valoración académica, tiene un alto grado de pertinencia.

Tabla 3

Percepción sobre listado de competencias

\begin{tabular}{lcc}
\hline & sí no & no \\
\hline 1. Son congruentes con el modelo pedagógico de la Universidad Nacional & $78 \%$ & $11 \%$
\end{tabular}

2 De acuerdo con ese listado de competencias, las tendencias de la profesión son emergentes. $\quad 89 \% \quad 0 \%$

3. Existe relación entre los elementos del perfil y la misión de la Escuela de Secretariado $\quad 33 \% \quad 44 \%$ Profesional.

4. Resaltan los principios filosóficos, epistemológicos y psicopedagógicos de la Universidad $\quad 33 \% \quad 44 \%$ Nacional.

5. Evidencian la incorporación de los avances científicos y tecnológicos en las áreas $\quad$ 89\% $\quad 0 \%$ profesionales involucradas.

6. Con esos rasgos, se podrá conseguir empleo una persona graduada $\quad 78 \% \quad 11 \%$

7. Los rasgos responden a las nuevas exigencias del mercado laboral. $\quad 78 \% \quad 11 \%$

8. Los rasgos responden a los de una persona formada integralmente para la sociedad actual. $\quad 67 \% \quad 22 \%$

9. Describe los dominios de competencias (grupos de capacidades y tareas esenciales $\quad 67 \% \quad 22 \%$ relacionadas que deben ser desplegadas por el futuro profesional).

10. Cuáles aspectos en la formación integral de un profesional en Educación Comercial, le agregaría usted:

Anote aquí:

Notas:

-Resultados de validación, promedios porcentuales según elección de la muestra.

-En la pregunta 10, la muestra anotó las siguientes competencias: dominio integral de uno o dos idiomas extranjeros, capacidad de desaprender y poder, con propiedad, reconstruir teoría desde la práctica, valores, responsabilidad, principios éticos, puntualidad y proactividad.

En la tabla 4 se detalla la valoración del perfil, según las académicas y los académicos consultados por su experiencia y conocimiento del contexto de la carrera de Licenciatura en Educación Comercial de la Escuela de Secretariado Profesional y del modelo por competencias. En su mayoría, están de acuerdo con los rasgos que considera el perfil. Indican que hay congruencia con el modelo pedagógico de la Universidad Nacional; que es una profesión emergente; que se evidencia la incorporación de los avances científicos y tecnológicos en los 
dominios del futuro profesional, que tiene posibilidades de empleabilidad porque responde a las exigencias del mercado laboral; que los rasgos responden a las necesidades de una formación integral tanto en los ámbitos pedagógico y disciplinar como de desarrollo humano.

En razón de lo anterior, la Propuesta de Perfil por Competencias para el Profesional en Educación Comercial cuenta con elementos básicos para orientar un proceso de rediseño curricular en el modelo por competencias, en el ámbito de la Escuela de Secretariado Profesional.

\section{Recomendaciones para su puesta en práctica}

Los proyectos curriculares, en el modelo por competencias, se perfilan como una tendencia nacional y regional para hacer propuestas de renovación de la rutina tradicionalista del aprender contenidos en las aulas, de las que la persona estudiante sale graduada con muchas competencias pero poco competente, como lo afirma Guy LeBoterf, (2007).

Cuando se inicia un proceso orientado hacia un cambio tan vertiginoso como es el de la educación tradicional a una educación por competencias, se requiere y exige el compromiso de todos los individuos involucrados: administración, docencia, estudiantado, comunidad, empresas e instituciones contratantes y colaboradoras de la institución educativa. Es necesario reinventar la práctica general de la institución educativa.

El perfil por competencias se plantea como un insumo básico para la deliberación profunda a lo interno de la Unidad Académica en estudio y para sustentar la discusión y darle seguimiento a un rediseño curricular en este modelo pedagógico. El liderazgo administrativo y académico puede hacer la diferencia en involucrar la Unidad Académica dentro de esta perspectiva de desarrollo del currículo, puesto que entre las funciones a las que le reta la modernidad, está el atender los fines más esenciales de la educación universitaria y, específicamente, la visión y misión de la Escuela de Secretariado Profesional.

Algunos aspectos que se recomienda considerar en la discusión en torno a la implementación de un rediseño curricular en el modelo por competencias son:

- Emprender esta propuesta de innovación y de generación de cambio, pues, a pesar de ser un proceso lento y continuo, con mucha motivación se pueden aprovechar los recursos materiales y humanos.

- Incorporar la relación teoría práctica a partir del aprendizaje en alternancia, pasantías, prácticas profesionales e indagación en puestos relacionados con los futuros espacios ocupacionales.

- Dentro de las condiciones materiales, los recursos del aula virtual en la plataforma Moodle, la cual se potencia como una herramienta fundamental en procesos en que 
URL: http://www.una.ac.cr/educare

la persona estudiante debe indagar, realizar actividades en sitio y la persona docente debe dar seguimiento y orientación permanente.

- Aprovechamiento de las condiciones de capacitación permanente a nivel de programas, que ofrece la misma Universidad al personal académico y administrativo, como el de becas, motivando en forma equitativa la especialización y la capacitación.

- Aprovechamiento de las condiciones que plantea el Modelo Pedagógico, el cual se orienta a la innovación, a la calidad y a la mejora de los procesos de educativos; así como de la libertad de cátedra para el enriquecimiento de la práctica, con principios de liderazgo académico.

- Trabajar con mayor énfasis el enlace necesario entre las dos Unidades Académicas que administran y gestionan la carrera de Educación Comercial.

- En este sentido, es relevante generar procesos de coordinación interdisciplinaria para orientar esa área formativa dentro de un proyecto por competencias.

\section{Referencias}

Alfaro, M., Gamboa, A., Jiménez, S., Martín, J., Ramírez, A. y Vargas, M-C. (2008). Construcción del perfil profesional docente de sétimo año: Respuesta a una necesidad actual. Revista Electrónica Educare, 12(2), 31-45. Recuperado de: http://www.revistas.una.ac.cr/index. php/EDUCARE/article/view/1433

Araya, I.yVarela, K. (2011). Aplicación de una propuesta teórica y metodológica para la producción de documentos en el modelo por competencias: Un estudio cuasiexperimental. Revista Actualidades Investigativas en Educación, 11(1), 1-31. Recuperado de http://revista.inie. ucr.ac.cr/uploads/tx magazine/aplicacion-propuesta-teorica-metodologica-producciondocumentos-araya.pdf

Araya, M. I. (enero-junio, 2011). Competencias en educación: Ideas para el diseño curricular desde la deliberación práctica y crítica, basada en el desarrollo humano y la transformación social. Revista Electrónica Educare, 15(1), 109-121. Recuperado de http://www.revistas. una.ac.cr/index.php/EDUCARE/article/view/884

Badilla, L. (s. f.). Documentos sobre algunos aportes al concepto de competencias desde la perspectiva de América Latina. Tuning América Latina. Recuperado de http://www.umex. org.mx/archivos/ACERVO/Tuning.pdf

Beneitone, P., Esquetini, C., González, J., Maletá, M. M., Siufi, G. y Wagenaar, R. (2007). Reflexiones y perspectivas de la Educación Superior en América Latina (Informe final -Proyecto Tuning- América Latina 2004-2007). Recuperado de http://es.scribd.com/doc/28967263/ Proyecto-Tuning-America-latina 
Bolívar, A. (2000). El currículum como un ámbito de estudio. En J. M. Escudero (Coord.), Diseño, desarrollo e innovación del currículum (pp. 23-44). Madrid: Síntesis.

Bozu, Z. (septiembre, 2007) El perfil de las competencias profesionales del profesorado de la ESO. PREAL. Recuperado de: http:www.oei.es/docentes/articulos/perfil competencias profesionales profesorado eso bozu.pdf.

Catalán, E. (julio, 2007). Conversando con Guy LeBoterf sobre el enfoque de competencias profesionales en el ámbito universitario (Trad. M. B. Peluffo). Sociedad y Conocimiento, 8, 44-46. Recuperado de http://www.faceaucentral.cl/pdf/socycon8.pdf

Delors, J. (1996). La Educación encierra un tesoro. Informe a la UNESCO de la Comisión Internacional sobre la Educación para el Siglo XXI. París: Ediciones UNESCO. Recuperado de http://www. unesco.org/education/pdf/DELORS S.PDF

Dirección General del Servicio Civil de Costa Rica. (2009). Manual descriptivo de clases de puestos docentes. Resolución DG-234-2009 publicada en La Gaceta Diario Oficial 158 Aviso 013-SC del 14/08/2009 con rige 01/07/2009. http://www.sercivil.go.cr/dgsc/area cd.php

Gimeno, J. (1996). El curriculum: Una reflexión sobre la práctica. España: Ediciones Morata.

Gimeno, J. (2009). Diez tesis sobre la aparente utilidad de las competencias en educación. En J. Gimeno (Coord.), Educar por competencias, ¿qué hay de nuevo? (pp. 15-58). Madrid: Morata.

Hawes, G. y Corvalán, O. (2005). Construcción de un perfil profesional (Proyecto Mecesup Tal 0101). Chile: Instituto de Investigación y Desarrollo Educacional. Universidad de Talca. Recuperado de: http://www.iide.cl/medios/iide/publicaciones/revistas/Construccion de un Perfil Profesional.pdf

Hernández, G. (1998). Paradigmas en psicología de la educación. México: Paidós.

Hernández, A. (2004). Capítulo V Perfil Profesional. Pedagogía Universitaria, 9(2), 69-98.

House, E. R. (1997). Evaluación, ética y poder. Madrid: Ediciones Morata.

Kemmis, S. (1998). El curriculum: Más allá de la teoría de la reproducción. Madrid: Morata.

Lévy-Levoyer, C. (1997). Gestión de las competencias, cómo analizarlas, cómo evaluarlas, cómo desarrollarlas. Barcelona: Gestión 2000.

Martínez, J. B. (2009). La ciudadanía se convierte en competencia: Avances y retrocesos. En J. Gimeno (Coord.), Educar por competencias, ¿qué hay de nuevo? (pp. 103-142). Madrid: Morata.

Mertens, L. (2000). La gestión por competencia laboral en la empresa y la formación profesional. España: OEl. Recuperado de http://www.oei.es/oeivirt/fp/iberfop01.pdf

Pérez, Á. I. (2009). ¿Competencias o pensamiento práctico?: La construcción de los significados de representación y de acción. En J. Gimeno (Coord.), Educar por competencias, ¿qué hay de nuevo? (pp. 59-102). Madrid: Morata. 
URL: http://www.una.ac.cr/educare

Perrenoud, P. (2007). Diez nuevas competencias para enseñar. Barcelona (5ª ed.). Barcelona: Graó.

Quesada, M. E., Cedeño, M. A. y Zamora, J. M. (2001). El diseño curricular en los planes de estudio: Aspectos teóricos y guía metodológica. Heredia. Costa Rica: EUNA.

Rial, A. (2007). Diseño curricular por competencias: El reto de la evaluación. Recuperado de http:// www.udg.edu/Portals/49/Docencia \%202010/Antonio Rial (text complementari).pdf

Ricoy-Lorenzo, M. C. y Pino-Juste, M. (2005). Estudio sobre el perfil del alumnado de Educación Social. ESE: Estudios sobre educación, 9, 127-143. Recuperado de http://dspace.unav.es/ dspace/bitstream/10171/8914/1/NC.PDF

Riesco, M. (2008). El enfoque por competencias en el EEES y sus implicaciones en la enseñanza y el aprendizaje. Tendencias Pedagógicas 13, 79-105. Recuperado de http://www.industriales. upct.es/pdfs/competencias riesco.pdf

Rodríguez, M. E. (2010). El perfil del docente de Matemática: Visión desde la triada matemáticacotidianidad y pedagogía integral. Revista Electrónica Actualidades Investigativas en Educación, 10(3), 1-19. Recuperado de http://revista.inie.ucr.ac.cr/ediciones/controlador/ Article/accion/show/articulo/el-perfil-del-docente-de-matematica-vision-desde-latriada-matematica-cotidianidad-y-pedagogia-integral.html

Tobón, S. (2005). Formación basada en competencias, pensamiento complejo, diseño curricular y didáctica ( $2^{\mathrm{a}}$ ed.). Bogotá. Colombia: Ecoe Ediciones.

Torres, J. (1998). Globalización e interdisciplinariedad: El curriculum integrado (3ª ed.). Madrid. España: Morata.

Torres, J. (2009). Obviando el debate sobre la cultura en el sistema educativo: Cómo ser competentes sin conocimientos. En J. Gimeno (Coord.), Educar por competencias, ¿qué hay de nuevo? (pp. 143-175). Madrid: Morata.

Universidad Nacional de Costa Rica, Escuela de Secretariado. (2007). Plan Estratégico 2007-2011. (Consejo de Académicos, Sesión 02-2007, 13 de marzo de 2007 según acuerdo ESPAC-195-2007). Heredia, Costa Rica: Autor.

\section{Cómo citar este artículo, según APA:}

Araya, I. (2012). Construyendo el perfil por competencias para el profesional en Educación Comercial. Revista Electrónica Educare, 16(3), 203-226. Consultado de http://www.revistas.una.ac.cr/index.php/EDUCARE/issue/current

Nota: Para citar este artículo en otros sistemas puede consultar el hipervínculo "Como citar el artículo" en la barra derecha de nuestro sitio web:

http://www.revistas.una.ac.cr/index.php/EDUCARE/index 